\title{
Tests of Micro-Pattern Gaseous Detectors for Active Target Time Projection Chambers in nuclear physics
}

\author{
J.Pancin ${ }^{\mathrm{a}, *}$, S. Damoy ${ }^{\mathrm{a}}$, D. Perez Loureiro ${ }^{\mathrm{a}}$, V. Chambert $^{\mathrm{b}}$, F. \\ Dorangeville $^{\mathrm{b}}$, F. Druillole ${ }^{\mathrm{c}}$, G.F. Grinyer ${ }^{\mathrm{a}}$, A. Lermitage ${ }^{\mathrm{b}}$, A. Maroni ${ }^{\mathrm{b}}$, G.

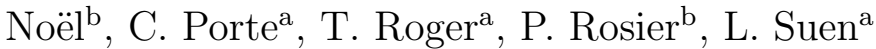 \\ ${ }^{a}$ GANIL, CEA/DSM-CNRS/IN2P3, Bvd H. Becquerel, Caen, France \\ ${ }^{b} I P N O, C N R S / I N 2 P 3$, Orsay, France \\ ${ }^{c} C E A, D S M / I r f u / S E D I$, Gif-Sur-Yvette, France
}

\begin{abstract}
Active target detection systems, where the gas used as the detection medium is also a target for nuclear reactions, have been used for a wide variety of nuclear physics applications since the eighties. Improvements in MPGD (Micro Pattern Gaseous Detectors) and in micro-electronics achieved in the last decade permit the development of a new generation of active targets with higher granularity pad planes that allow spatial and time information to be determined with unprecedented accuracy. A novel active target and time projection chamber (ACTAR TPC), that will be used to study reactions and decays of exotic nuclei at facilities such as SPIRAL2, is presently under development and will be based on MPGD technology. Several MPGD (Micromegas and Thick GEM) coupled to a $2 \times 2 \mathrm{~mm}^{2}$ pixellated pad plane have been tested and their performances have been determined with different gases over a wide range of pressures. Of particular interest for nuclear physics experiments are the angular and energy resolutions. The angular resolution has been determined to be better than $1^{\circ} \mathrm{FWHM}$ for short traces of about $4 \mathrm{~cm}$ in length and the energy resolution deduced from the particle range was found to be better than $5 \%$ for $5.5 \mathrm{MeV} \alpha$ particles. These performances have been compared to Geant4 simulations. These experimental results validate the use of these detectors for several applications in nuclear physics.

Keywords: Active Target, Time Projection Chamber, Micromegas, Thick GEM
\end{abstract}

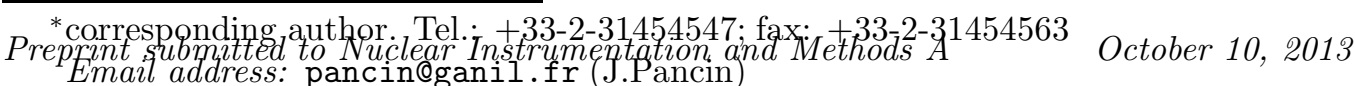




\section{Introduction}

With the ongoing improvements in radioactive ion beam production at several facilities worldwide, new possibilities will soon be available for studying the structure and decays of the most "exotic" nuclei, which are those furthest from the line of beta stability [1]. The intensity of the most exotic beams available remains however usually low. In this regard, the use of active targets has become an attractive alternative to study the most exotic nuclei. This type of detection setup, where the detection medium is also used as a target presents several advantages. It allows the simultaneous detection and identification of low-energy recoils that would stop in a classical solid target. The effective target thickness can thus be increased (by adjusting the pressure) to study nuclei produced at the lowest intensities, or to study reactions with very negative Q-values where the recoils are emitted with low energies. Active targets designed to study specific types of reactions are already in existence. The IKAR active target [2] has been used to study the matter distribution of very exotic light ions through proton inelastic scattering. The CENBG TPC [3] is used to provide the three-dimensional reconstruction of two-proton radioactivity events and was used to prove the existence of this type of decay in ${ }^{45} \mathrm{Fe}$. Other active targets like MAYA at GANIL [4] have been built for more general use. With a solid angle coverage of about $2 \pi$, MAYA has been used for the study of transfer reactions with very exotic beams $[5,6,7]$ or giant resonances [8] in radioactive $\mathrm{Ni}$ isotopes.

With the upcoming availability of fission fragment beams at SPIRAL2, there is an obvious need for active targets with higher dynamic range in order to study, for example, the evolution of shell structure around the neutron number $\mathrm{N}=50$ and $\mathrm{N}=82$ magic numbers via single neutron transfer reactions. Higher granularity and higher counting-rate capabilities will permit the study of giant resonances and key reactions for those nuclei situated in, or near, the astrophysical rapid neutron and rapid-proton capture processes [9]. In this framework, based on the concept of the active target MAYA, the more efficient and versatile ACTAR TPC (ACtive TARget and Time Projection Chamber) is being developed. This detector will consist of a gas-filled volume of approximately $25 \times 25 \times 20 \mathrm{~cm}^{3}$. As in the MAYA active target, the ionization electrons produced along the charged particles tracks, i.e. the beam or the charged recoils produced in the reactions of interest between projectiles with the gas atoms, drift under the influence of an electric field to an amplification gap. The latter will consist of either Micromegas [10] (as used 
in the AT-TPC at MSU [11]) or ThGEM (Thick GEM) [12, 13], chosen for their robustness and high-rate counting purposes. The amplification system will be coupled to a high granularity pad plane with $2 \times 2 \mathrm{~mm}^{2}$ pixels, which will allow events to be reconstructed with good angular resolution even for short track lengths and with an excellent spatial resolution on the stopping points and hence a good energy resolution.

Regarding the foreseen geometry, several validation tests have been performed using one ThGEM of 600 microns (with $0.4 \mathrm{~mm}$ diameter holes and $0.7 \mathrm{~mm}$ pitch) and two Micromegas detectors of 128 and 256 microns amplification gaps, respectively. These tests consisted of several angular resolution and stopping-point measurements using a pixellated pad plane with $2 \times 2$ $\mathrm{mm}^{2}$ pads. The setup consisted of an $\alpha$ source and a silicon strip detector to select $\alpha$ trajectories above the pad plane. The entire ensemble was installed in the existing drift-field cage of MAYA. Gases at different pressures $\left(\mathrm{He}+\mathrm{CF}_{4}(2 \%)\right.$ from 400 to 800 mbar and $\mathrm{iC}_{4} \mathrm{H}_{10}$ from 25 to 75 mbar) were used. Another gas, $\mathrm{Ar}+\mathrm{CF}_{4}(2 \%)$, was used to stop the alpha-particles over the pad plane and determine the energy and range resolutions.

In section 2 and 3, the experimental set-up and the data analysis are presented in detail. Section 4 is devoted to the results of angular resolution with the different conditions of gas and pressure. The stopping point measurements are described in section 5. Experimental results are then compared to simulations in section 6 .

\section{Experimental set-up}

The prototype MPGDs were mounted on a circular PCB pad plane of 5.6 $\mathrm{cm}$ diameter with square pads of $2 \mathrm{~mm}$ side length that totaled 576 channels. As only 288 channels could be read using a single AFTER card (electronics previously developed for the T2K experiment [14]), only a fraction of the total pads could be connected while all others were grounded. The AFTER card was placed either below the pad plane in the gas or outside the chamber depending on the thermal conductivity of the gas. Two bulk Micromegas [15] were tested on this pad plane, one with an amplification gap of 128 $\mu \mathrm{m}$ and the other with $256 \mu \mathrm{m}$. A ThGEM foil of $600 \mu \mathrm{m}$ thick was also tested and was positioned at a height of $2 \mathrm{~mm}$ above of the pad plane. The detection system was inserted at the bottom of the MAYA drift field cage and was surrounded by a copper plate that was biased at the micromesh or the ThGEM voltage to maintain the homogeneity of the electric drift 
field. The field cage is composed of printed circuit board with copper strips (with $3 \mathrm{~mm}$ pitch) on the front and side panels and a wire plane on the back panel to allow particles to escape [4]. As shown in figure 1, a DSSSD (Double Sided Silicon Strip Detector) with 16 channels on each side and a strip pitch of $3.12 \mathrm{~mm}$ was placed at the end of the chamber. A mask with 16 slits of $10 \times 0.6 \mathrm{~mm}^{2}$ was positioned in front of the DSSSD. A mixed alpha source (3 alpha-particles with energies of $5.1 \mathrm{MeV}, 5.5 \mathrm{MeV}$ and $5.8 \mathrm{MeV}$ from ${ }^{239} \mathrm{Pu},{ }^{241} \mathrm{Am}$ and ${ }^{244} \mathrm{Cm}$, respectively) was inserted at a distance of 184 $\mathrm{mm}$ from the Si detector and at a height of $10 \mathrm{~cm}$ above the MPGD pad plane. In the horizontal direction, the source was $13 \mathrm{~mm}$ from the start of the active area of the detector. The source has a diameter of $5 \mathrm{~mm}$ and can be collimated. The data acquisition system was triggered by the detection of an alpha-particle in the Si detector and the charge signals on the pad plane were used to reconstruct the alpha trajectory. The slits of the Si mask were positioned either vertically or horizontally depending on the desired angular resolution measurement.

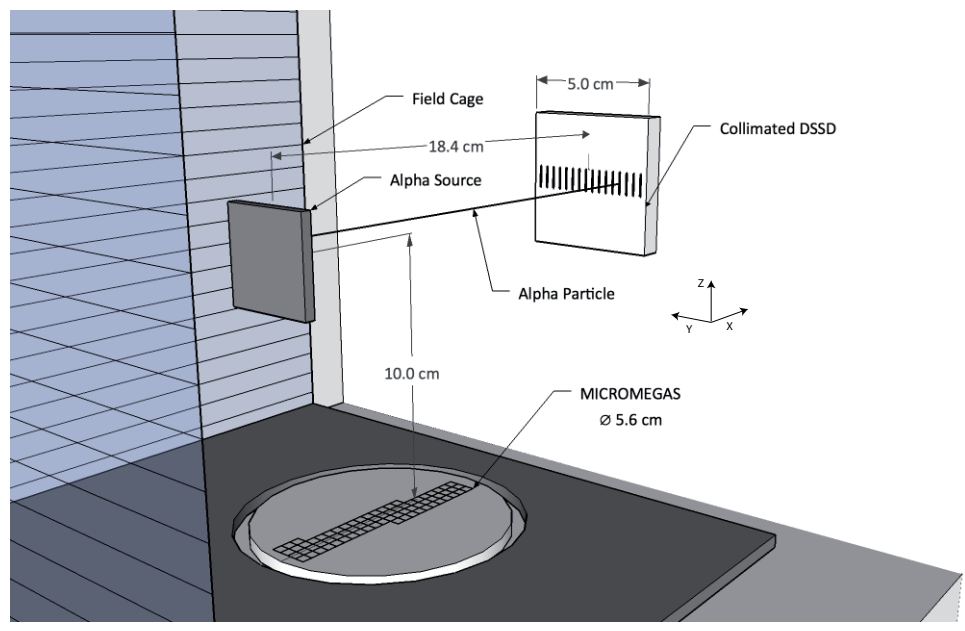

Figure 1: Schematic view of the complete setup for horizontal measurements. For vertical measurements the DSSSD and the mask were rotated by $90^{\circ}$.

The filling gas used in the chamber was supplied through a gas regulation system that ensured a constant flow and pressure. The $128 \mu \mathrm{m}$ Micromegas prototype was tested in $\mathrm{He}+\mathrm{CF}_{4}(2 \%)$ at 500, 600 and 800 mbar whereas the $256 \mu \mathrm{m}$ detector was tested at 400, 500 and 600 mbar and in pure $\mathrm{iC}_{4} \mathrm{H}_{10}$ at 25, 50 and 70 mbar. The ThGEM was only tested in pure $\mathrm{iC}_{4} \mathrm{H}_{10}$ at 
25, 50 and 75 mbar. The maximum pressures (800 mbar in the He mixture and 75 mbar in isobutane) were chosen so that the alpha particles could reach the silicon detector. The lower pressures were adapted to the different amplification systems and their own sparking limits and gain properties. For this reason, the $256 \mu \mathrm{m}$ Micromegas was prefered to the $128 \mu \mathrm{m}$ in isobutane [16]. The values of voltages are specified for each result given later in this article. For Micromegas, they are given as: $\mathrm{V}_{\text {mesh }} / \mathrm{V}_{\text {drift }}$ with $\mathrm{V}_{\text {mesh }}$ the micromesh voltage and $\mathrm{V}_{\text {drift }}$ the voltage applied to the drift cathode. For the ThGEM, they are given as: $\mathrm{V}_{\text {down }} / \mathrm{V}_{\text {up }} / \mathrm{V}_{\text {drift }}$ with $\mathrm{V}_{\text {down }}$ the voltage applied to the bottom electrode in front of the pad plane (generally called extraction voltage) and $\mathrm{V}_{\text {up }}$ the voltage applied to the top electrode.

\section{Data analysis and trace reconstruction}

Figure 2.a shows a schematic view of how the pads were connected to the electronics card (288 total channels from sectors 1,2,3 and 4). The AFTER card is equipped with one preamplifier and shaping amplifier per channel. Signals are sampled at a maximum rate of $100 \mathrm{MHz}$ and registered in a 12 bit ADC on trigger request. Parameters such as the dynamic range, the shaping time and the sampling rate can all be modified in software. In general, a shaping time of $400 \mathrm{~ns}$ and a sampling rate of $100 \mathrm{MHz}$ were used. The dynamic range was typically $120 \mathrm{fC}$ but could be increased to 240 or $360 \mathrm{fC}$ if the detector gain or the energy deposition was too high. The homogeneity of the pad response was studied by injecting a pulser on the mesh of the Micromegas detector and it was found to be better than $2 \%$. The analysis program utilises the main characteristics of the event-by-event signals such as the channel number, the baseline and the noise (standard deviation of the baseline), the signal amplitude and the times (start, maximum and stop times). Only those channels whose total collected charge exceeded a specified threshold were recorded. This threshold was typically chosen to be 10 times the noise level. The mean standard deviation of the baseline (noise level) was around $4 \mathrm{ADC}$ channels (for short cables of less than $10 \mathrm{~cm}$ length and a dynamic range of $120 \mathrm{fC}$ ) which corresponds to less than 800 electrons. An example of a 2D histogram with the signal amplitude of the channels versus their $\mathrm{X}$ and $\mathrm{Y}$ spatial coordinates for a single projected trace is shown in figure 2.b. Due to the influence of the transverse diffusion of the electrons in the drift gap, the traces have a transverse multiplicity that is larger than a single pad. The start time of each pad was calculated using a software CFD 
162

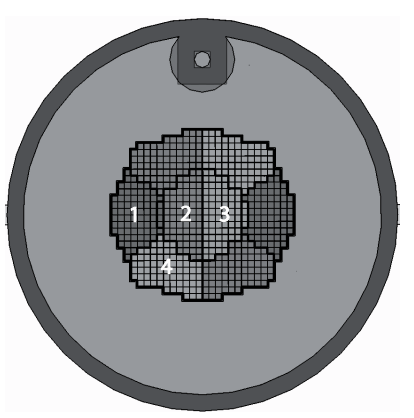

(a)

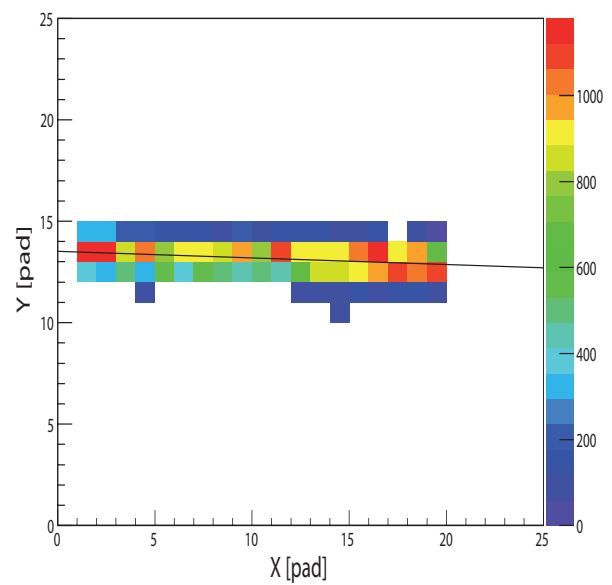

(b)

Figure 2: (a) The pad plane was divided into eight sectors of 36 pixels of $2 \times 2 \mathrm{~mm}^{2}$ and sectors 1,2,3 and 4 were connected to a single 288 channels AFTER card. (b) Representative $2 \mathrm{D}$ histogram of the charge signal amplitude in ADC values using Micromegas versus the $\mathrm{X}$ and $\mathrm{Y}$ spatial coordinates and resulting best-fit trajectory (solid line).

A two-step track reconstruction analysis was performed on an event-byevent basis. The horizontal angle of the projected 2D alpha trace was first calculated using a linear fit. The vertical angle was then evaluated using the time differences between the pads along the trajectory. The horizontal measurement is absolute, the real position of the projected track is reconstructed and it was not necessary to collimate the source. However, the vertical angle measurement is relative. The angle is calculated but the absolute height of the particle is not known. It was therefore necessary in this case to use a collimated source.

For horizontal angles, the trajectories are determined from a $\chi^{2}$ minimization between the fit and the pad centers in the $\mathrm{X}$ and $\mathrm{Y}$ directions weighted by their individual collected charge [17]. The result is a straight line that gives the direction and the origin of the track as plotted in figure 2.b. Once the straight line parameters (slope and origin) are known, the final Y coordinate of the track at the position of the Si detector can be extrapolated using the known physical geometry of the setup. One can then obtain the angle considering a point-like source. From the connected sectors shown in 
figure 2.a, the maximum track length corresponds to the first 3 sectors of the detector. This implies that the linear fits could be performed using charge distributions with a maximum length of $4.2 \mathrm{~cm}(3$ sectors with 7 pads of 2 mm length per sector). In practice, the first and last rows of the pad planes were removed from the analysis to remove observed edge effects. Resolutions were thus calculated using a maximum trace length of $3.8 \mathrm{~cm}$. For comparison, MAYA pads are $8.9 \mathrm{~mm}$ length and traces of at least $5 \mathrm{~cm}$ in length are required to deduce the horizontal direction.

For vertical angles, the setup restricted traces to angles that were primarily parallel to the X-axis (horizontal angles $\theta_{h}<7^{\circ}$ for the most external silicon strips). The traces were divided into pad rows and it was the start time of the pads with the maximum charge in that particular row that was used in the analysis. This minimizes the influence of transverse diffusion of the ionization electrons on the time determination. A linear fit of the start time of each row versus the row was then performed. From these fits, and using the drift speed of the gas obtained from GARFIELD simulations [18], the relative vertical angles $\theta_{v}$ of the traces can be determined.

\section{Angular resolution results}

Four slits of the Si detector out of the 16 total were used to trigger the electronics. Well separated slits such as 1, 5, 9 and 13 were used to increase the total counting rate and ensured the distributions could be resolved. Plots of the reconstructed slit positions were obtained (figure 3) and the angular resolution was deduced using a gaussian fit to the collected distribution of each slit. The contribution to the total resolution from the slits themselves was less than $0.2^{\circ}$ (FWHM) and was negligible compared to the overall resolutions obtained. Results of the angular resolution measurements (in FWHM) are provided in figure 4 . The results shown are the average values of the widths of the four reconstructed slits. This corresponds to angles between $\pm 7^{\circ}$ in the horizontal and the vertical directions.

The first set of measurements were performed in $\mathrm{He}+\mathrm{CF}_{4}(2 \%)$ using the two Micromegas detectors at several pressures (figure 4.a). Voltages of $190 /-2000,-210 /-2000$ and $-250 /-2000 \mathrm{~V}$ were applied to the $128 \mu \mathrm{m}$ detector for 500, 600 and 800 mbar of pressure, respectively. Voltages of $-250 /-2000$, $-260 /-2000$ and $-270 /-2000 \mathrm{~V}$ were applied to the $256 \mu \mathrm{m}$ detector for 400 , 500 and 600 mbar of pressure, respectively. The horizontal resolutions are of order $1^{\circ}$ and are comparable between the two detectors. This confirms that 


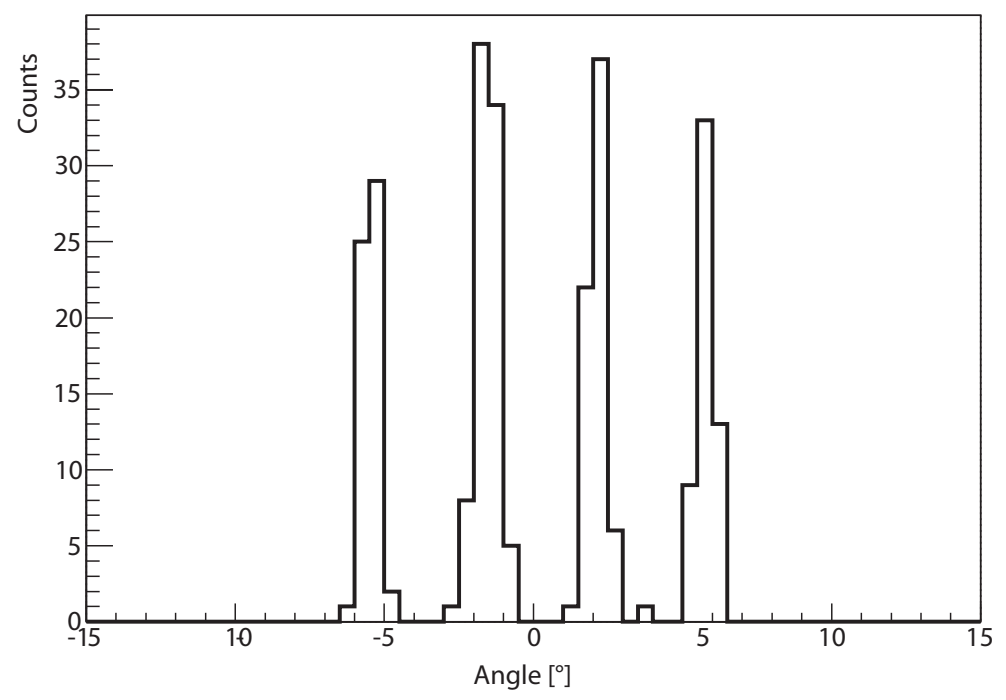

Figure 3: Histogram of the slit reconstruction in degrees.

the slit widths can be neglected. The mean transverse multiplicities were 3.5 pads for both detectors. This is larger than what would be expected from the amplification gap alone and is thus a result of the electron diffusion in the drift gap. From GARFIELD simulations, a strong variation of the transverse diffusion coefficient is not expected for pressures between 500 mbar and 800 mbar (about $400 \mu \mathrm{m} / \sqrt{c m}$ with $10 \%$ variation). The transverse multiplicity can increase to $\sim 4$ pads in certain conditions (higher gains for example) but this has a negligible influence on the angular resolution for trace lengths exceeding $3 \mathrm{~cm}$. Straggling effects calculated with TRIM [19] for a trace length of $184 \mathrm{~mm}$ and with $5.5 \mathrm{MeV}$ alpha particles are also indicated in figure 4. The long distance of $184 \mathrm{~mm}$ between the source and the Si detector is clearly a drawback of the present set-up. Each event is affected by straggling along this entire length and not just the $3.8 \mathrm{~cm}$ that the detector is sensitive to. The slight degradation of the horizontal resolution with increasing pressure is consistent with straggling. The measured angular resolutions are however bigger than the effect of straggling given by TRIM. The straggling given by TRIM could be underestimated and this could explain the difference between straggling curves and measurements. Since the horizontal angular reconstruction relies on the individual charge of the pads, the angular resolution could also be degraded compared to straggling because of the energy 
resolution of the pads (see section 6).

Variations in the vertical resolution are similarly influenced by straggling. However, as explained above, the drift time is relative between channels. The exact heights of the alpha particles are unknown and thus only their angles can be deduced. It was therefore necessary to restrict the emission angles of the source using a 2-mm diameter collimator that corresponds to a broadening of the vertical angular distribution of $0.5^{\circ} \mathrm{FWHM}$. The time resolution of the electronics and the CFD method has been estimated to $7 \mathrm{~ns}$ FWHM by injecting pulses on the micromesh and reading the corresponding signals on the pads. For a drift speed of $1 \mathrm{~cm} / \mu \mathrm{s}$ and taking into the account the number of pads used in the vertical angle analysis, the time resolution of the electronics leads to an additional angular uncertainty of $\sim 0.1^{\circ}$. The overall vertical resolution of $\sim 1^{\circ}$ is thus primarily limited from straggling effects and the diameter of the collimator.

Figure 4.b shows complementary results to figure 4.a but for low pressures of pure isobutane for the $256 \mu \mathrm{m}$ Micromegas detector and the ThGEM. Voltages of $-380 /-2000,-410 /-2200$ and $-380 /-2500 \mathrm{~V}$ were applied to the $256 \mu \mathrm{m}$ detector for 25,50 and 75 mbar of pressure, respectively (the voltage at 75 mbar was kept lower to avoid the saturation of the electronics). The voltages of the ThGEM were -200/-700/-1700, -60/-700/-1700, and -350/1050/-2500 for 25, 50 and 75 mbar of pressure, respectively. The results are also compared to straggling calculations from TRIM. Angular resolutions are comparable to $\mathrm{He}+\mathrm{CF}_{4}(2 \%)$ and are equivalent between the two detector types. However, vertical angular resolutions (close to $1.3^{\circ}$ ) at low pressure are slightly degraded relative to the horizontal resolutions due (partially) to the increased drift speed of the gas. The estimated uncertainty of the time resolution of the electronics with a drift speed of $5 \mathrm{~cm} / \mu$ s in isobutane leads to a vertical angular uncertainty of $\sim 0.5^{\circ}$. This is five times larger than the corresponding uncertainty in the helium mixture at higher pressure. However, the time uncertainty is not sufficient to explain the degradation of the vertical angle resolution at low pressures and it is probable that this time uncertainty has been under evaluated (see section 6).

The influence of the total trace length on the resulting angular resolution is particularly important for the reconstruction of low-energy particles. For example, a $100 \mathrm{keV}$ proton will have a range of less than $8 \mathrm{~mm}$ in $50 \mathrm{mbar}$ of isobutane. Short trace-length events were investigated by removing data in software from the $3.8 \mathrm{~cm}$ tracks in successive intervals of $0.4 \mathrm{~cm}$ (2 pads) and the analysis described above was repeated. Angular resolutions (horizontal 


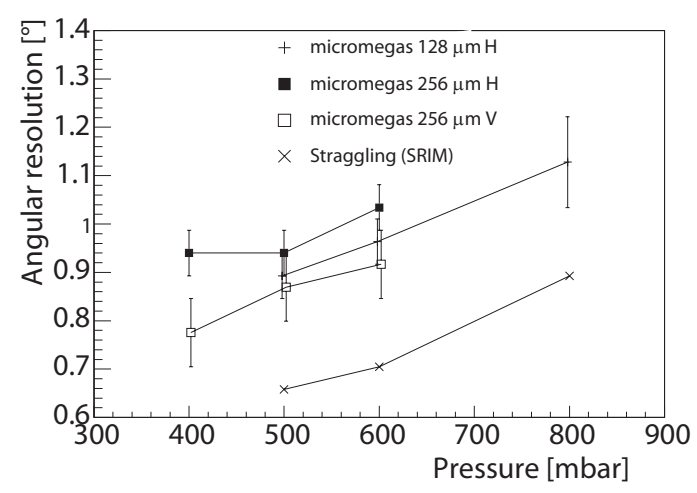

(a)

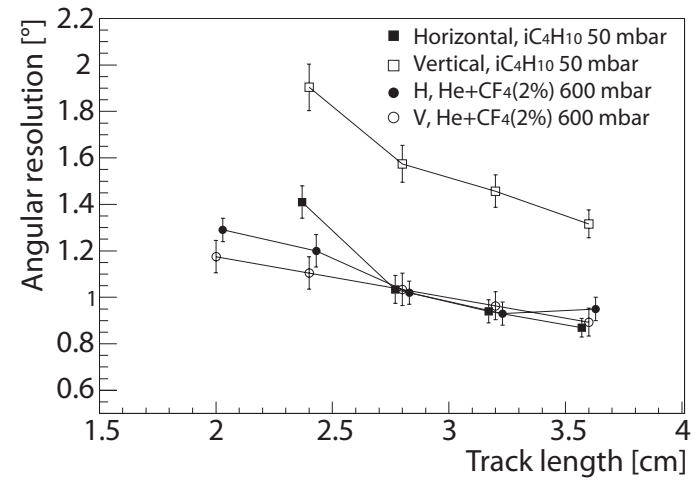

(c)

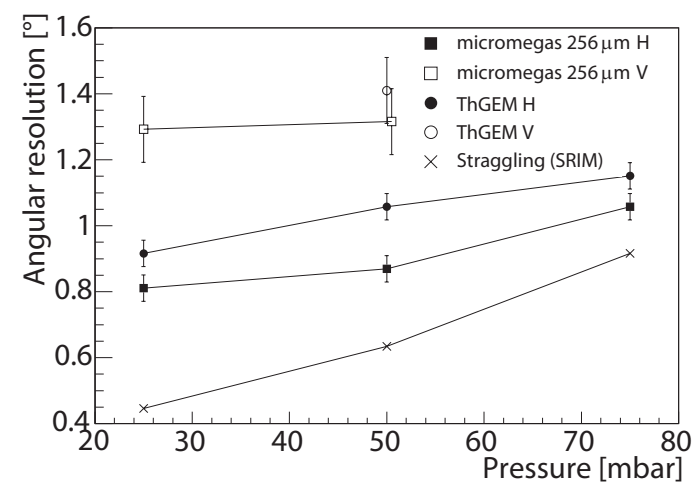

(b)

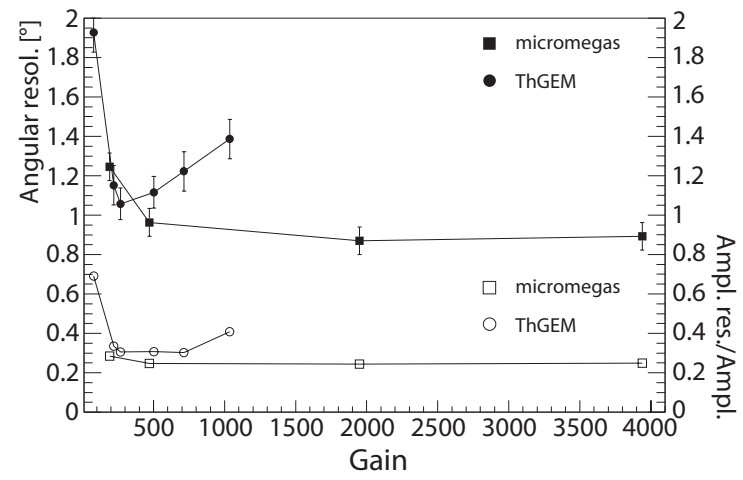

(d)

Figure 4: Experimental angular resolution results. (a) Angular resolution (in FWHM) in $\mathrm{He}+\mathrm{CF}_{4}(2 \%)$ (H for horizontal and $\mathrm{V}$ for vertical) for the two Micromegas detectors, (b) angular resolution in $\mathrm{iC}_{4} \mathrm{H}_{10}$ for the $256 \mu \mathrm{m}$ Micromegas and the ThGEM, (c) angular resolution versus track length for the $256 \mu \mathrm{m}$ Micromegas detector in $\mathrm{He}+\mathrm{CF}_{4}(2 \%)$ at 600 mbar and $\mathrm{iC}_{4} \mathrm{H}_{10}$ at 50 mbar, and (d) angular resolution (solid symbols, axis on the left) and the energy resolution (open symbols, axis on the right) versus the amplification gain in $\mathrm{iC}_{4} \mathrm{H}_{10}$ at 50 mbar. 
and vertical) versus the trace length are provided in figure 4.c for the 256 $\mu \mathrm{m}$ Micromegas in 50 mbar of $\mathrm{iC}_{4} \mathrm{H}_{10}$ and $\mathrm{He}+\mathrm{CF}_{4}(2 \%)$ at 600 mbar. A degradation of the resolution in both horizontal and vertical directions with the track length is observed in both gases. For trace lengths larger than 2.5 $\mathrm{cm}$, angular resolutions are comparable with the exception of the vertical resolution in low-pressure isobutane. As described above, this is primarily due to the high drift speed at low pressure coupled with the time resolution of the electronics. Vertical resolutions follow the trend imposed by the coupling of this time resolution and the track length (number of pads used for the time fitting). The degradation of the horizontal resolution for shorter track lengths can have two origins. Besides the straggling effects inherent to the setup, the ratio between the track length and the transverse multiplicity has a direct influence on the accuracy of the straight line fit used to reconstruct the direction of the tracks. Gases with low transverse diffusion coefficients should be used when events with short ranges must be reconstructed or, at least, since the choice of the gas and pressure are imposed by the nuclear reaction, the drift voltage or the quencher percentage should be adapted to minimize the transverse diffusion. However, the mean transverse multiplicity must be larger than 2 pads to keep a good angular resolution in every direction.

Measurements with different detector gains were performed in 50 mbar of pure isobutane with the $256 \mu \mathrm{m}$ Micromegas and the ThGEM detectors and results are shown in figure 4.d. The Micromegas voltage was varied from $-300 /-2000$ to $-420 /-2000 \mathrm{~V}$. The THGEM voltages were varied from $-20 /-660 /-1500$ to $-190 /-830 /-1700 \mathrm{~V}$ keeping a constant difference between $\mathrm{V}_{\text {down }}$ and $\mathrm{V}_{\text {up }}$. It was not the gain of the foil that was varied but rather the extraction field (the field applied between the bottom of the ThGEM and the pad plane). Angular resolutions and energy resolutions are plotted versus the amplification gain and both show similar trends. The horizontal angle reconstruction is weighted by the amplitude of the signal collected on the individual pads. The energy resolution therefore influences the determination of the reconstructed trajectory. The energy resolution of $22 \%$ (FWHM), for gains higher than 500 in the Micromegas detector, was calculated from the dispersion of the mean amplitudes on several rows of the pad plane. It includes the variations in the number of electrons produced during the ionization and the avalanche processes, the electronic noise contribution and the energy straggling of the alphas, which is the main contribution at high gains. The energy deposition of the alpha-particles was $\sim 25 \mathrm{keV} /$ pad in $\mathrm{iC}_{4} \mathrm{H}_{10}$ at 50 mbar. Amplification gains were calculated using the mean 


$\begin{array}{cccc}\text { Radioactive species } & \text { Alpha energies } & \text { SRIM Range } & \text { LISE Straggling } \\ { }^{239} \mathrm{Pu} & 5.15 \mathrm{MeV} & 36.5 \mathrm{~mm} & 0.35 \mathrm{~mm} \\ { }^{241} \mathrm{Am} & 5.48 \mathrm{MeV} & 40 \mathrm{~mm} & 0.4 \mathrm{~mm} \\ { }^{244} \mathrm{Cm} & 5.8 \mathrm{MeV} & 43.5 \mathrm{~mm} & 0.45 \mathrm{~mm}\end{array}$

Table 1: Characteristics of the $3 \alpha$ source, ranges and associated straggling in $\mathrm{Ar}+\mathrm{CF}_{4}(2 \%)$ at 1100 mbar.

signal amplitude over several rows, the charge dynamic range of the electronic channels, the average energy deposited and the pair energy creation $(\sim 20$ $\mathrm{eV}$ ). The maximum gain of the ThGEM is lower than the Micromegas at 50 mbar but the ThGEM used was probably too thin for this pressure.

\section{Stopping point measurements}

To obtain Bragg-peak events with $5.5 \mathrm{MeV} \alpha$ particles within the $4.2 \mathrm{~cm}$ active length of the detectors, Ar gas with $2 \% \mathrm{CF}_{4}$ at 1100 mbar was employed. In table 1, energies and ranges of the 3 main alphas of the source are summarized for this particular gas mixture. Range studies were performed using the $256 \mu \mathrm{m}$ Micromegas detector with a voltage of -350/-3350 V.

The micromesh signal, rather than the Si detector, was used as trigger for the electronics card. Reconstruction of the individual tracks on the pad plane were performed using the analysis techniques described above. An example of the amplitude of the signals on the pads projected in the transverse direction with respect to the alpha trace is presented in figure 5. The resulting Bragg peak is in good agreement with SRIM calculations. However, the energy loss at the beginning of the trace is larger than expected and is likely due to edge effects in the electrostatic field of the drift region.

Bragg-peak distributions were fitted on an event-by-event basis using cubic spline interpolation. The maxima were deduced, and the ranges determined using the position where the amplitude equals one fourth of the maximum. This is an empirical method that depends upon the particular detector and the diffusion of the gas, but it is sufficiently accurate in this context. Once the projected length is determined, the horizontal and vertical angles allow the total track length to be deduced in 3 dimensions. Figure 6 shows the calculated end points of several reconstructed $\alpha$-particles. A clear separation of the 3 alpha energies confirms the accuracy of this analysis procedure. 


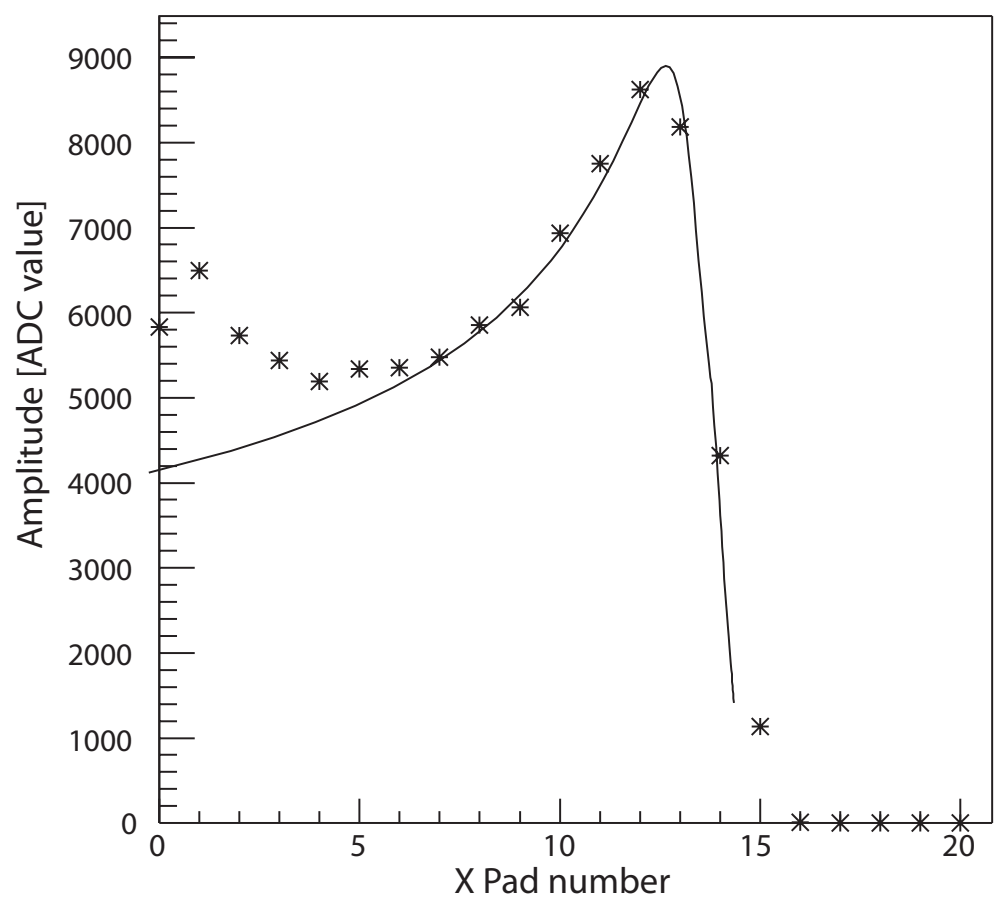

Figure 5: Amplitude signals versus track length and expected distribution from SRIM for a $5.5 \mathrm{MeV}$ alpha in 1100 mbar of $\mathrm{Ar}+\mathrm{CF}_{4}(5 \%)$. 


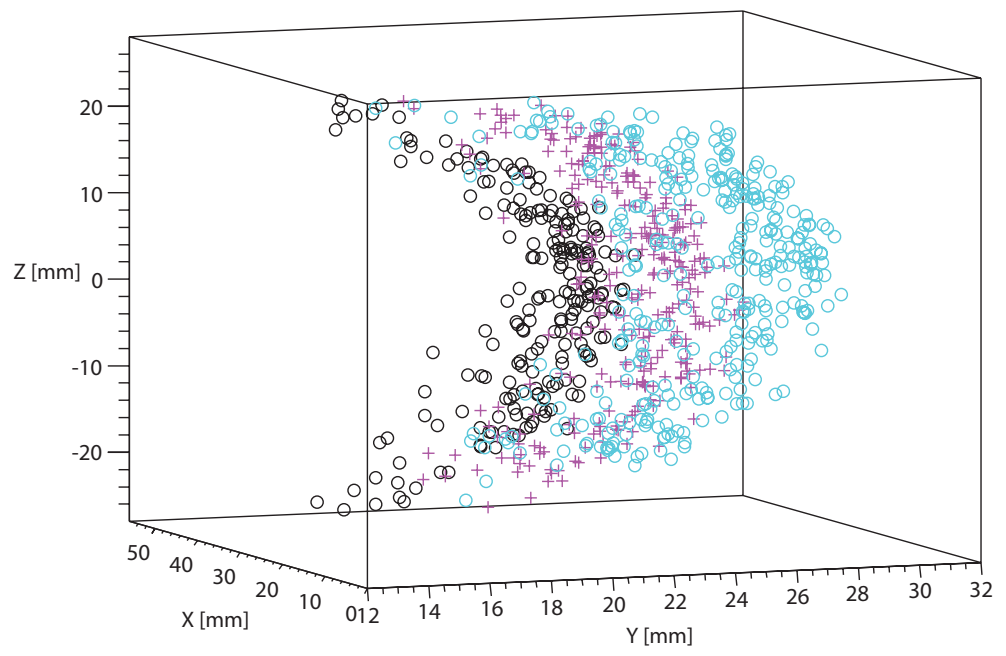

Figure 6: Plot of the reconstructed ranges for the $3 \alpha$-particles (black $\mathrm{Pu}$, red Am and blue $\mathrm{Cm}$ ).

Amplitude and range resolutions obtained at angles restricted to $\pm 10^{\circ}$ in vertical and $\pm 5^{\circ}$ in horizontal are shown in figure 7 and the 3 peaks are very well separated. From the total amplitude of the signals registered on the pads, the energy resolution obtained for these three peaks (from lowest to highest energy) are 5\%,4.5\% and 6\% (FWHM) respectively. The energy resolution is degraded for the highest alpha energy. Its stopping point was located between two sectors of the pad plane and thus two different AFTER chips. This resolution could be improved with a better calibration of the system. The reconstructed ranges are $36.7 \pm 0.4 \mathrm{~mm}, 39.9 \pm 0.4 \mathrm{~mm}$ and $43.3 \pm 0.4 \mathrm{~mm}$ for the 3 peaks, respectively. These values are in excellent agreement with the SRIM values shown in table 1 . The range resolution on the stopping points are $2.4 \%, 2.3 \%$ and $2.2 \%$ (FWHM), respectively. In terms of energy resolution, a $2.5 \%$ FWHM range corresponds to $2 \%$ for a $5.8 \mathrm{MeV} \alpha$-particle. The detector behaves as expected and the energy resolution is consistent with results obtained in [20].

Charge and range resolutions were also obtained when the angular restriction is removed taking into account the set-up geometry $\left( \pm 50^{\circ}\right.$ in vertical and $\pm 18^{\circ}$ in horizontal) and results are shown in figure 8 for the charge (a) and range (b) measurements respectively. From the signal amplitudes, the en- 


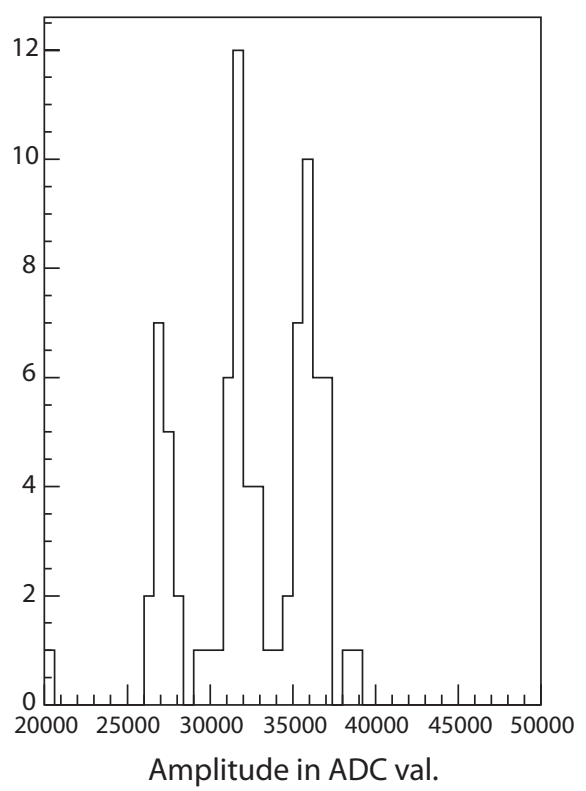

(a)

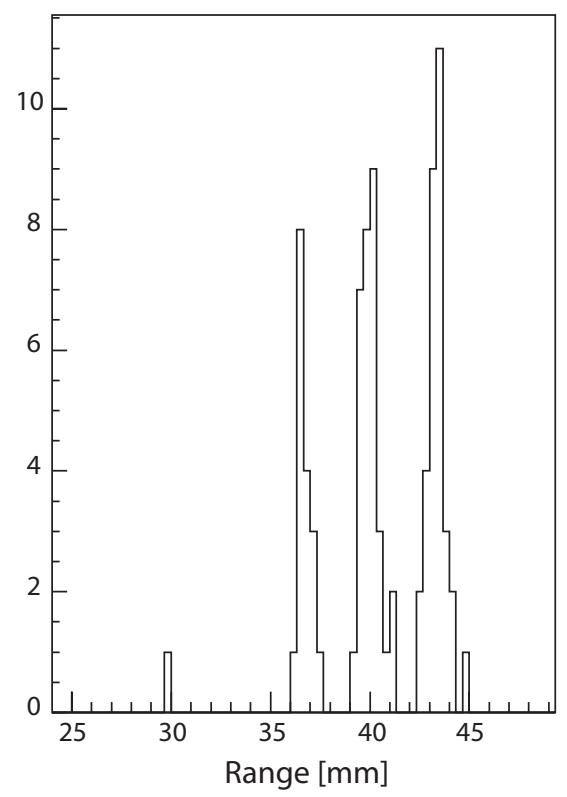

(b)

Figure 7: Signal amplitude (a) and range (b) resolutions at closed angles. 
ergy resolution is degraded relative to the results at restricted angles and is likely due to the distance between the source and the active pad plane that is more significant for larger angles. In terms of the total range, the resolution on the trace lengths (from lowest to highest energy) are $4.6 \%, 5.5 \%$ and $4.27 \%$ (FWHM), respectively. The reconstructed ranges are $36.8 \pm 0.7 \mathrm{~mm}$, $40.2 \pm 0.9 \mathrm{~mm}$ and $43.7 \pm 0.8 \mathrm{~mm}$ for the 3 peaks. The energy resolution from the range analysis is, to a large extent, preserved since the $5 \%$ FWHM range corresponds to $4 \%$ in energy resolution for a $5.8 \mathrm{MeV} \alpha$-particle.

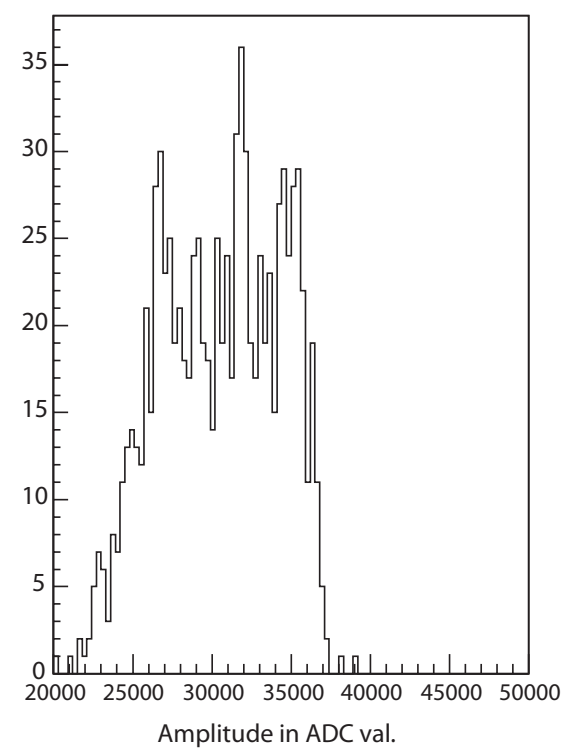

(a)

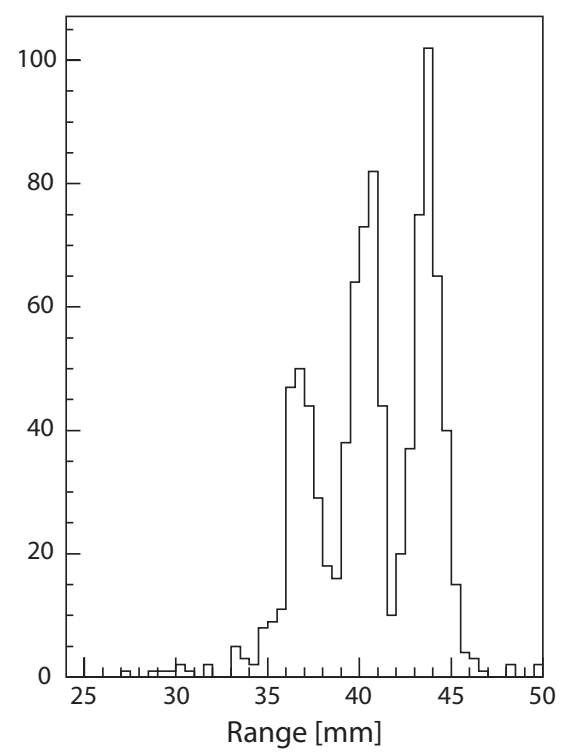

(b)

Figure 8: Signal amplitude (a) and track length (b) resolutions at open angles.

\section{Simulations}

The complete experimental setup was simulated using a dedicated program based on the ROOT data analysis framework [21] and the Geant4 toolkit [22]. The program employed GEANT4 to describe the interactions of the particles with the atoms or molecules of the gas and for the determination of the energy deposited at each interaction position along their trajectories. The precise physical geometry of the setup including the $\mathrm{Si}$ strip detector that was used as the trigger detector in the experiment (for 
the angular resolution measurements) were also defined in Geant4. Because the ionization electrons produced along the trajectories of the $\alpha$ particles, their transportation through the drift gap under an applied electric field, electron amplification in the Micromegas, and the subsequent charge collection on the position-sensitive pad plane cannot be specified in Geant4, it was necessary to include these processes within a set of macros developed in ROOT. A description of these processes, how they were combined with the GEANT4 simulated energy deposition and position information, and comparison between the simulation and the experimental results are presented below.

\subsection{Gas ionization and detector response}

The ionization of the gas and the drift of the resulting electrons were treated as follows. At each Geant4 interaction point, the mean number of electrons was calculated from the ratio between the energy deposited and the average energy required to produce an electron-ion pair ( $\mathrm{W} \sim 30 \mathrm{eV}$ ) [23]. Fluctuations to this mean number are then included using a Poisson distribution. In order to reproduce the charge spread that arises from diffusion along the vertical drift length (considering an ideal electrostatic drift field), the arrival point of the electrons at the amplification plane was obtained using a Gaussian randomization of the horizontal coordinates with respect to the initial interaction point. The width of this Gaussian distribution is given by $\sqrt{2 D h / v}$ where $D$ is the diffusion coefficient of the gas, $h$ the vertical height of the interaction point with respect to the pad plane and $v$ the electron drift velocity. The parametrization of each gas was calculated using MAGBOLTZ [24] that uses as inputs the gas species, pressure, and the electric field applied across the drift region. Pressures used in the simulations were the same as for the experiment: 600 mbar for $\mathrm{He}+\mathrm{CF}_{4}(2 \%), 50 \mathrm{mbar}$ for $\left.\mathrm{iC}_{4} \mathrm{H}_{10}\right)$ and 1100 mbar of $\mathrm{Ar}+\mathrm{CF}_{4}(2 \%)$ for range determinations. The time required for the electrons to reach the anode (the time projection) was obtained at each position using the (vertical) position coordinate and the constant drift velocity in the gas (with a drift electric field of $100 \mathrm{~V} / \mathrm{cm}$ ). A time resolution of $3 \mathrm{~ns}$ (corresponding to 1 standard deviation) was added according to the experimental results.

The microscopic details of the avalanche process in the Micromegas were not included in the simulations. Instead, an overall multiplicative gain factor was applied to every electron that was selected with a probability according to a Polya distribution $[25,26]$. Fluctuations on this gain, that arise from 
the statistical nature of the avalanche, are therefore intrinsically included with this approach $(\theta=2.2$ was chosen for the Polya parameter [26]). For an entire simulated $\alpha$ particle trajectory, which corresponds to many Geant 4 single-interaction points, the total charge collected on each pad was obtained from the sum of all of the individual electron contributions to that pad. A software threshold of 8000 electrons was then applied to the simulated data to be consistent with the experimental results described above in Sec. 3. No additional effects (such as noise) were included in the simulations. The charge resolution obtained with this method was $\sim 35 \%$ (FWHM). This value is larger than the experimental resolution given in figure 4 .d (which was $\sim 22 \%$ ) indicating that the energy straggling in Geant4 is likely overestimated.

\subsection{Angular resolution simulation results}

To distinguish between the relative contributions of straggling effects in the experimental results obtained for the angular resolution, two different simulations were performed. The first used the identical physical geometry and analysis methods as employed in the experiment. The Si detector was placed a total distance of $184 \mathrm{~mm}$ from the $\alpha$ source and results were obtained from fits applied to only the first few cm of each simulated trajectory. The angular resolutions obtained from these simulations are shown in figure 9 (squares) for both gases versus the length of the fitted trace. Qualitatively, the trends observed in the simulated results are in excellent agreement with with experimental data. The simulated data reproduce both the increased horizontal resolution for short traces and the difference between the vertical angular resolution between the helium and isobutane gases (open squares) that arise from differences in their drift velocities as described above. It was even possible to show that a time resolution degraded to 6 ns (sigma, instead of $3 \mathrm{~ns}$ ) was enough to reproduce the difference between the helium mixture and the isobutane in vertical angular resolution (see figure 4.c). Quantitatively, the simulated results are systematically lower than the experimental data of figure 4 by $\sim 20 \%$. It should be emphasized that the angular resolutions are completely dominated by lateral straggling of the particles in the gas. In the simulations, these effects are described by Geant4 libraries that were found to be consistent with SRIM calculations. The decreased resolution observed experimentally therefore indicates that lateral straggling effects are likely underestimated in Geant4 and SRIM. This has been observed previously in Ref. [27], for example, where precise measurements of lateral straggling from a highly-collimated beam of protons and $\alpha$ particles 
(at energies similar to those in our study) were also underestimated in SRIM by approximately $40 \%$.

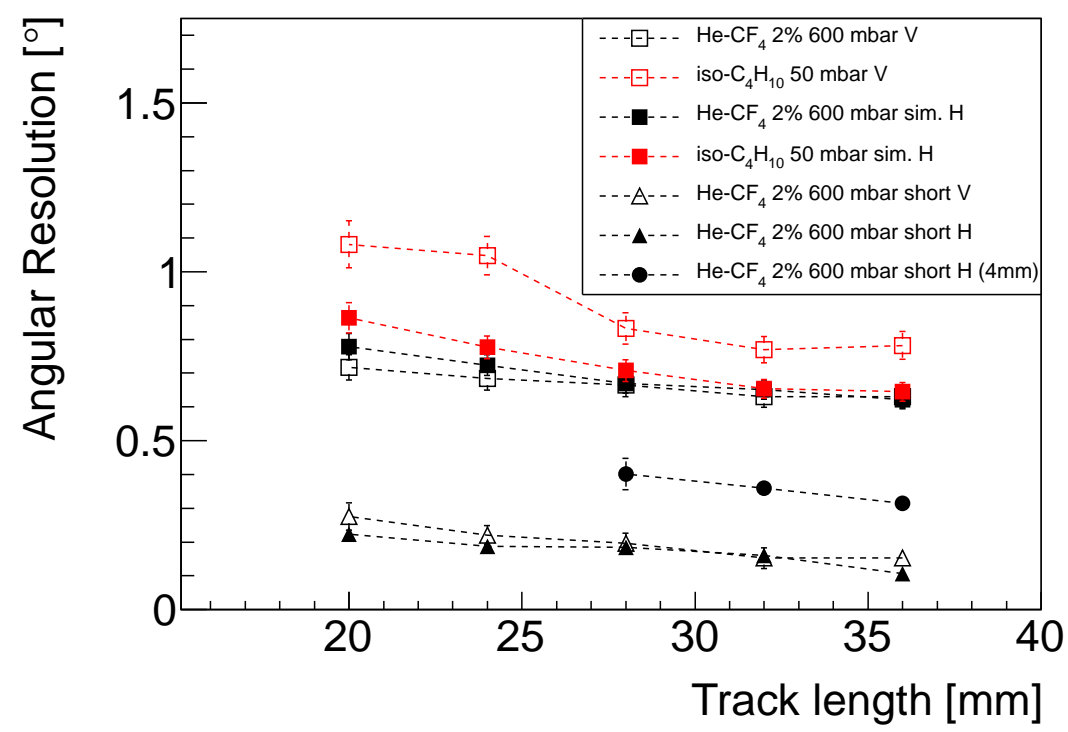

Figure 9: Results of the simulation based on the experimental setup: Angular resolutions versus track length in horizontal and vertical for the helium mixture and the isobutane.

In order to minimize these angular straggling effects, a second set of simulations were performed with the Si positioned closer to the source. In this case, we did not analyse only a small portion of a longer trace, but instead fitted the entire trace length obtained with a reduced source-to-Si distance that was varied for every simulation at the different distances shown in Fig. 9. To ensure that the results of this simulation were consistent with the previous ones, we had to account for the geometrical difference between the two configurations as placing the slit closer to the source increases the overall range of emission angles for $\alpha$ particles that can reach the detector. As shown in Fig. 9, the angular resolution results obtained from this second set of simulations (triangles) are significantly better than the previous values due to the decrease in lateral straggling along these much shorter trajectories. This analysis provides further confirmation for the dominance of lateral straggling in the angular resolution that can be achieved. Moreover, it confirms that the energy resolution is not the main contribution to angular resolution in our measurements (as long as the gain is high enough, see figure 4.d). 
The same simulations were performed using a $4 \mathrm{~mm}$ pad size for the digitization and the results are represented by circles in Fig. 9. The angular resolution obtained is a factor two larger compared to the $2 \mathrm{~mm}$ pad. In addition, with the lower transverse multiplicity for a bigger pad size, the fitting algorithm cannot always converged in the track reconstruction for traces shorter than $28 \mathrm{~mm}$.

\section{3. range simulation results}

The simulation was also used for the study of the range of the $\alpha$-particles in the detector. The method for the determination of the range is the same as was used with the experimental data: the projected range is calculated from the Bragg curve and then corrected by the horizontal and vertical angles obtained from the fits of the charge distribution on the pad plane and the drift times, respectively. Figure 10 shows the distribution of the ranges of the $\alpha$-particles for the different energies. The left panel corresponds to the distribution obtained selecting forward angles $\left( \pm 5^{\circ}\right.$ and $\pm 10^{\circ}$ in horizontal and vertical, respectively). The mean values obtained for the reconstructed ranges for closed angles are $35.9 \pm 0.4 \mathrm{~mm}, 39.3 \pm 0.4 \mathrm{~mm}$ and $42.8 \pm 0.5 \mathrm{~mm}$ for $5.15 \mathrm{MeV}, 5.48 \mathrm{MeV}$ and $5.8 \mathrm{MeV}$, respectively. The range resolution on the stopping point is then $2.5 \% \mathrm{FWHM}$. The mean values obtained are in very good agreement with SRIM range calculations as well as the experimental results. The simulated range resolution is also in good agreement with the one obtained experimentally. The right panel of Fig. 10, represents the range distribution for a wider angular range $\left( \pm 30^{\circ}\right.$ in horizontal and $\pm 50^{\circ}$ in vertical). We observe that the range resolution is still good enough to resolve the three peaks of the alpha source with resolutions of $4.2 \%(35.6 \pm 0.6 \mathrm{~mm})$, $4.2 \%(39.0 \pm 0.7 \mathrm{~mm})$ and $3.4 \%(42.4 \pm 0.6 \mathrm{~mm})$.

\section{Conclusion}

Several tests were performed using Micromegas and ThGEM detectors coupled to a $2 \times 2 \mathrm{~mm}^{2}$ pad plane in a TPC. The angular resolutions and the accuracy of the stopping point reconstructions were investigated as part of a preliminary study into the possible use of these detectors for low-energy nuclear physics applications. Angular resolutions better than $1^{\circ} \mathrm{FWHM}$ (in both the horizontal and vertical directions) were obtained in different gases and at various pressures with both detector types. The influence of the gain was investigated and it was shown that the best angular resolutions were 

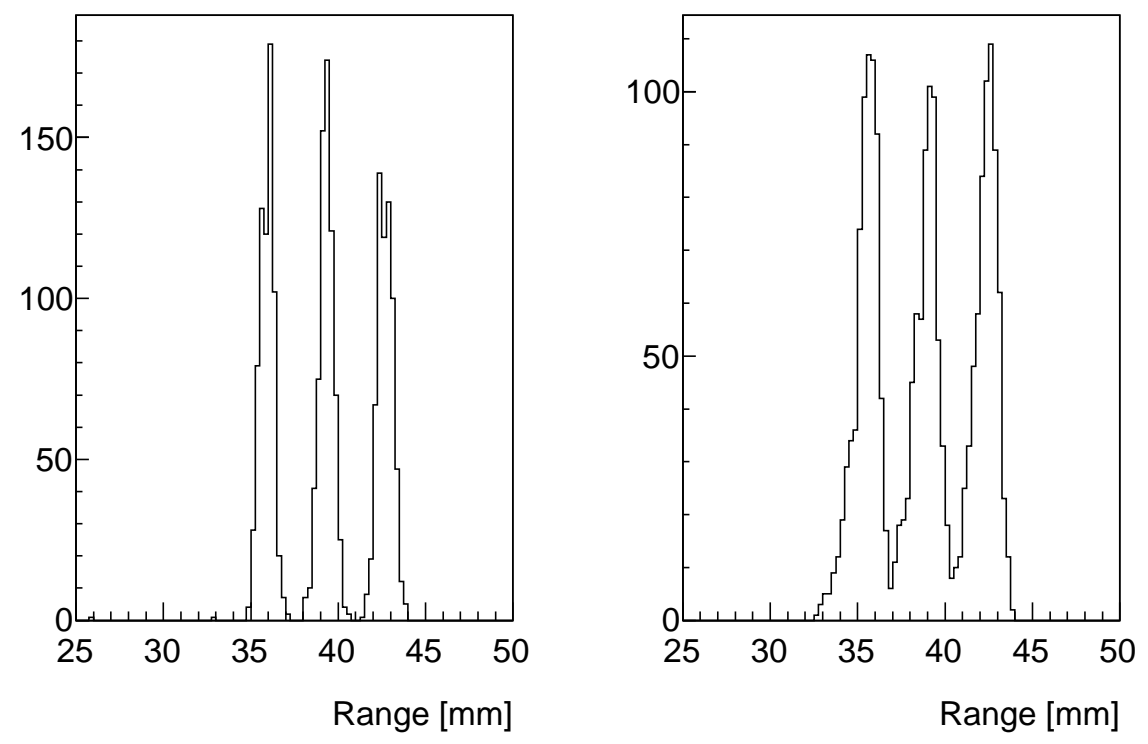

Figure 10: Range distribution calculated from the simulation: closed angle (left) and open angle (right).

obtained when the energy resolution is optimized, generally at intermediate gains. An energy resolution of $5 \%$ FWHM for $5.8 \mathrm{MeV} \alpha$-particles was measured from the charge profile and, using the range measurements, can be improved to $4 \%$ FWHM. All of these experimental results have been described with a Geant4 simulation and it has been shown that care must be taken before determining any quantitative conclusions from such simulations since their description of the angular and energy straggling processes appear to be under and over-estimated, respectively. The experimental results presented in this work are better than present detectors such as MAYA and they validate the use of Micromegas and ThGEMs for active-target applications in nuclear physics where high density pad planes are required. These results also validate the use of a high granularity pad plane $\left(2 \times 2 \mathrm{~mm}^{2}\right)$ with MPGDs since the transverse diffusion in the drift gap ensures a good transverse multiplicity between 3 and 4 pads.

A demonstrator Micromegas version of the ACTAR TPC detection system with 2048 pixels is presently under construction and will be used to test a new set of electronics for TPCs (GET: General Electronics for TPCs 
funded by France ANR-09-BLAN60203-01) that are being developed for such high-density applications. The demonstrator will provide an opportunity to address other challenges that can arise with big chambers such as the drift field homogeneity, and the mechanical integration and the robustness of the connections.

\section{Acknowledgments}

The research leading to these results has received funding from the European Union Seventh Framework Program (FP7/2007-2013) under grant agreement nos. 212692 and 283745. We would like to thank the SEDI laboratory from CEA/DSM/IRFU in Saclay for the Micromegas bulk detectors. Particular thanks go also to the electronic workshop of IPN from CNRS/IN2P3 in Orsay.

\section{References}

[1] O. Sorlin, M.-G. Porquet, Nuclear magic numbers: New features far from stability, Progress in Particle and Nuclear Physics, 61-2(2008)602673.

[2] A.A. Vorobyov et al., experimental apparatus for the study of small angle neutron-proton elastic scattering at intermediate energies, Nucl. Instr. and Meth. A $\mathbf{2 7 0 ( 1 9 8 8 ) 4 1 9 . ~}$

[3] B. Blank et al., A time projection chamber for three-dimensional reconstruction of two-proton radioactivity events, Nucl. Instr. and Meth. A $\mathbf{6 1 3}(2010) 65$.

[4] CH.-E. Demonchy et al., MAYA: An active-target detector for binary reactions with exotic beams, Nucl. Instr. and Meth. A 583(2007)341.

[5] M. Caam̃ano et al., Resonance state in ${ }^{7} H$, Phys Rev. Lett. $\mathbf{9 9}(2007) 062502$.

[6] I. Tanihata et al., Measurement of the two-halo neutron transfer reaction in ${ }^{1} H\left({ }^{11} \mathrm{Li},{ }^{9} \mathrm{Li}\right)^{3} \mathrm{H}$ at $3 \mathrm{~A} . \mathrm{MeV}$, Phys Rev. Lett. 100(2009)192502.

[7] T. Roger et al., Mass of ${ }^{11} \mathrm{Li}$ from the ${ }^{1} \mathrm{H}\left({ }^{11} \mathrm{Li},{ }^{9} \mathrm{Li}\right)^{3} \mathrm{H}$ reaction, Phys Rev. C. 79(2009)031603. 
[8] C. Monrozeau et al., First Measurement of the Giant Monopole and Quadrupole Resonances in a Short-Lived Nucleus: ${ }^{56} \mathrm{Ni}$, Phys. Rev. Lett. 100(2008)042501.

[9] A. Schatz, Nuclear Astrophysics with Rare Isotopes,Nucl. Phys. A $\mathbf{8 2 7}(2009) 26 \mathrm{c}$.

[10] I. Giomataris et al., Development and prospect of the new gaseous detector Micromegas, Nucl. Instr. and Meth. A 419(1998)239.

[11] D. Suzuki et al., Prototype AT-TPC: Toward a new generation active target time projection chamber for radioactive beam experiments, Nucl. Instr. and Meth. A 691(2012)39-54.

[12] C. Shalem et al., Advances in Thick GEM-like gaseous electron multipliers-Part I: atmospheric pressure operation, Nucl. Instr. and Meth. A 558(2006)475.

[13] C. Shalem et al., Advances in Thick GEM-like gaseous electron multipliers-Part II: Low pressure operation, Nucl. Instr. and Meth. A $\mathbf{5 5 8 ( 2 0 0 6 ) 4 6 8 . ~}$

[14] P. Baron et al, AFTER, an ASIC for the readout of the large T2K time projection chambers,IEEE transactions on Nuclear Science 55$\mathbf{3}(2008) 1174$.

[15] I. Giomataris et al., Micromegas in a bulk, Nucl. Instr. and Meth. A $\mathbf{5 6 0}(2006) 405$.

[16] M. Nakhostin et al., Performance of a low-pressure Micromegas-like gaseous detector, Nucl. Instr. and Meth. A 598-2(2009)496.

[17] T. Roger et al., Tracking algorithms for the active target MAYA, Nucl. Instr. and Meth. A 638(2011)134.

[18] R. Veenhof, http://garfield.web.cern.ch/garfield/, 1984.

[19] J. Ziegler, http://www.srim.org/, 1983.

[20] F.J. Iguaz et al, Micromegas detector developments for Dark Matter directional detection with MIMAC,JINST6P07002(2011). 
[21] http://root.cern.ch

[22] S. Agostinelli et al., GEANT4 - A simulation toolkit, Nucl. Instr. and Meth. A 506(2003)250.

[23] G. F. Knoll, Radiation detection and Measurement, John Wiley and Sons, Inc., New York 2000.

[24] S. Biagi, Monte Carlo simulation of electron drift and diffusion in counting gases under the influence of electric and magnetic fields, Nucl. Instr. and Meth. A421(1999)234-240.

[25] J. Derré et al., Fast signal and single electron detection with a MICROMEGAS photodetector, Nucl. Instr. and Meth. A 449(2001)314.

[26] T. Zerguerras et al.,Single-electron response and energy resolution of a Micromegas detector, Nucl. Instr. and Meth. A 608(2009)397.

[27] C. Michelet et al, Measurement of lateral straggling using a microbeam,Nucl. Instr. and Meth. B181(2001)157-163. 